\title{
Active recovery training does not affect the antioxidant response to soccer games in elite female players
}

\author{
Helena Andersson ${ }^{1}$, Anette Karlsen ${ }^{2}$, Rune Blomhoff ${ }^{2}$, Truls Raastad ${ }^{3}$ and Fawzi Kadi ${ }^{1 *}$ \\ ${ }^{1}$ School of Health and Medical Sciences, Örebro University, 70182 Örebro, Sweden \\ ${ }^{2}$ Department of Nutrition, Institute of Basic Medical Sciences, Faculty of Medicine, University of Oslo, Oslo, Norway \\ ${ }^{3}$ Norwegian School of Sport Sciences, Oslo, Norway
}

(Received 2 February 2010 - Revised 17 May 2010 - Accepted 18 May 2010 - First published online 8 July 2010)

Changes in plasma endogenous and dietary antioxidants and oxidative stress markers were studied following two 90 min elite female soccer games separated by $72 \mathrm{~h}$ of either active or passive recovery. The active recovery group $(n 8)$ trained for $1 \mathrm{~h}$ at 22 and $46 \mathrm{~h}$ after the first game (lowintensity cycling and resistance training), while the passive group rested $(n 8)$. Blood samples were taken before the games; immediately after the games; 21, 45 and $69 \mathrm{~h}$ after the first game; and immediately after the second game. The oxidative stress markers and antioxidants were not affected by active recovery. The oxidative stress marker GSSG increased by the same extent after both the games, while the lipid peroxidation marker diacron-reactive oxygen metabolite remained unchanged. The endogenous antioxidants total glutathione and uric acid and ferric reducing/ antioxidant power increased immediately after both the games with the same amplitude, while increases in cysteine, cysteine-glycine and total thiols reached significant levels only after the second game. The changes in dietary antioxidants after the first game were either rapid and persistent (tocopherols and ascorbic acid (AA) increased; polyphenols decreased) or delayed (carotenoids). This resulted in high pre-second game levels of tocopherols, AA and carotenoids. Polyphenols returned to baseline at $69 \mathrm{~h}$, and were not affected by the second game. In conclusion, the soccerassociated dietary antioxidant defence, but not the endogenous antioxidant defence, is persistent. Similar acute oxidative stress and endogenous antioxidant responses and dissimilar dietary antioxidant reactions occur during two repeated female soccer games. Finally, the complex antioxidant response to soccer is not affected by active recovery training.

Intermittent exercise: Training: Recovery: Free radicals: Football

Strenuous intermittent exercise increases the production of reactive oxygen species (ROS), which subsequently activates antioxidant defence mechanisms in order to maintain homoeostasis $^{(1)}$. Disturbances in the balance between ROS production and the capacity of the antioxidant defence in favour of ROS production may lead to oxidative stress ${ }^{(2)}$. Excessive exercise-induced oxidative stress is believed to be a factor leading to fatigue ${ }^{(3)}$, and may thereby contribute to decreased performance during exercise and possibly to a delayed recovery process.

The intensive physical work performed during a soccer game relies heavily on both aerobic and anaerobic energy production ${ }^{(4)}$. Several performance parameters, such as sprint, jump ability and isokinetic strength, are reported to be impaired following a soccer game in both male ${ }^{(5,6)}$ and female players ${ }^{(7)}$. Additionally, increased oxidative stress following a single soccer game and a soccer-specific running protocol has been reported in male players ${ }^{(5,6,8,9)}$. However, we have shown previously that a single soccer game induces a parallel elevation in GSSG and several antioxidant compounds without changes in lipid peroxidation in elite female players ${ }^{(10)}$. Whether the robust antioxidant response that occurs after a single soccer game in female players is maintained during repeated games is unknown. It is important to address this issue given the fact that international women soccer tournaments allow only for $2 \mathrm{~d}$ of recovery between games. Such a short recovery period between two games underlies the growing interest in developing effective recovery strategies to improve performance.

Various strategies including massage, contrast-water immersion and active recovery training are used by elite athletes ${ }^{(11)}$. Currently, no studies evaluating the effectiveness of active recovery training $1 \mathrm{~d}$ after a competitive soccer game are available. The theoretical benefits of active recovery training include an acceleration of the recovery time of neuromuscular and blood markers of physical stress and a quicker restoration of the redox balance and the inflammatory reaction. Accordingly, an efficient recovery would promote optimal competitive performance, and may help elite players to cope with high training and game loads ${ }^{(11,12)}$. In this respect, we have reported previously that active recovery does not accelerate neuromuscular parameters ${ }^{(7)}$ or affect the inflammatory mediators after elite female soccer games ${ }^{(13)}$. The effects of active recovery training on oxidative stress markers and antioxidants during repeated elite soccer games remain unknown.

Abbreviations: AA, ascorbic acid; CK, creatine kinase; d-ROM, diacron-reactive oxygen metabolite; ROS, reactive oxygen species. 
We have previously established the acute changes in plasma antioxidants and oxidative stress markers following a single soccer game. The aim of the present study was to compare the oxidative stress reaction and the response of endogenous and dietary antioxidants following two repeated female soccer games separated by $72 \mathrm{~h}$ of either passive or active recovery.

\section{Methods}

\section{Subjects}

Twenty-two elite female soccer players from the highest division in Sweden and Norway played two 90 min games separated by $72 \mathrm{~h}$. Three players were not available on the recovery days between the matches, and one player was taking medication (insulin). Moreover, as the physical loading of goalkeepers differs from that of the field players, they were not included in the analysis. Thus, in total, blood samples from sixteen field players (height: 167 (SE 5) cm, weight: 64 (SE 2) kg, age: 22 (SE 3) years, $\mathrm{V}_{\mathrm{O} 2 \max }: 54$ (SE 3) ml/kg per min) were used for the analysis of oxidative stress markers and antioxidant levels. The games were conducted in the middle of the soccer season when the players are accustomed to playing games. Before the commencement of the study, the players had rested at least $3 \mathrm{~d}$ from games and $2 \mathrm{~d}$ from soccer training. None of the players were smokers. None of the players reported the use of anti-inflammatory drugs or antioxidant supplements. Approximately, half of the players reported the use of oral contraceptives. The players who were not taking contraceptives had regular menstrual cycles. The menstrual cycle phase of the participants in the present study was not assessed. According to Akova et al. ${ }^{(14)}$, the phase of the menstrual cycle might influence some of the antioxidant/prooxidant markers. It is interesting to note that Chung et al. ${ }^{(15)}$ investigated the role of oestrogens in exercise-induced oxidative stress, and found minimal differences in oxidative stress levels between the luteal and follicular phases during exercise.

The players were informed about the experimental procedures and possible discomforts associated with the study, and they gave their written informed consent to participate. The study was conducted according to policy statement set forth in the Declaration of Helsinki, and was approved by the Regional Ethics Committee of Uppsala, Sweden.

\section{Experimental design}

Two games were played during a period of $4 \mathrm{~d}$. The players performed a standardised $20 \mathrm{~min}$ soccer-specific warm-up on the field before both the games. The games were separated by $2 \mathrm{~d}$ of either active or passive recovery. A randomised blocked design was used to assign the players into an active recovery group $(n 8)$ or a passive recovery group $(n 8)$. The groups were matched for age (23 (SE 4) years active and 22 (SE 3) years passive), height (167 (SE 6) cm active and 167 (SE 3) $\mathrm{cm}$ passive), weight $(63.3$ (SE 7.1) $\mathrm{kg}$ active and 65.0 (SE 4.9) kg passive), maximal oxygen consumption (55.4 (SE 3.6) $\mathrm{ml} / \mathrm{min}$ per $\mathrm{kg}$ active and 53.8 (SE 2.4) $\mathrm{ml} / \mathrm{min}$ per $\mathrm{kg}$ passive) and playing position. Blood was sampled before the games $(-3 \mathrm{~h})$; within $15-20 \mathrm{~min}$ of the games
$(0 \mathrm{~h}) ; 21,45$ and $69 \mathrm{~h}$ after the first game; and within $15-20 \mathrm{~min}$ of the second game $(74 \mathrm{~h})$. A standardised breakfast was planned before the blood sampling. The players had breakfast $1 \mathrm{~h}$ before the blood sampling. We noted similar weather conditions during both the games (light rain and approximately $12^{\circ} \mathrm{C}$ ). We have reported previously that the amount of high-intensity running (1.09 (SE 0.2) v. 1.11 (SE $0 \cdot 1) \mathrm{km}$ ), plasma creatine kinase (CK) levels (323 (SE 36) v. 376 (SE 53) U/l), neuromuscular changes and fluid intake were similar in both the games ${ }^{(7)}$. We have also reported previously that the mean heart rate was slightly higher $(P<0.05)$ in the second game $(168$ (SE 2) bpm) than in the first game $\left(162\right.$ (SE 2) bpm) ${ }^{(13)}$.

\section{Diet}

The food intake was standardised during the study period, starting on the evening before the game. Intake of carbohydrate, protein and fat was adjusted to the players' body weight $(55 / 60 / 65 / 70 \mathrm{~kg}$, respectively) to meet the recommendations for daily recovery (intake $\geq 6 \mathrm{~g} / \mathrm{kg}$ body weight carbohydrate and $\geq 1.2 \mathrm{~g} /$ body weight protein $)^{(16)}$. The food was chosen to replicate the players' normal diet as much as possible, and did not contain any food items with known high antioxidant levels (see Andersson et $a{ }^{(10)}$ for more details).

\section{Active recovery training}

The active recovery training consisted of $1 \mathrm{~h}$ of low-intensity exercise that was performed between the two games (22 and $46 \mathrm{~h}$ after the first game). The training consisted of $30 \mathrm{~min}$ sub-maximal cycling (60\% of peak heart rate) and $30 \mathrm{~min}$ low-intensity resistance training $(<50 \%$ of one repetition maximum). During the period between the two games, all the players were allowed to perform their habitual daily activities with the exception of participating in any kind of exercise. During the $1 \mathrm{~h}$ period when the active recovery group performed the low-intensity exercise, the control group was instructed to rest ${ }^{(7)}$.

\section{Biochemical measures for oxidative stress makers and antioxidant levels}

All analyses were performed using the blood samples collected from the antecubital vein. Plasma samples were analysed for GSH and GSSG as the markers of oxidative stress, and for GSH:GSSG ratio as an indicator of plasma pro-oxidant and antioxidant redox status ${ }^{(17)}$. Quantification of endogenous GSSG and GSH was performed using a twodimensional chromatographic system with parallel Hypercarb columns coupled with dual fluorescence detectors. The CV for the method was below $7 \%$. The ratio of GSH:GSSG was calculated by dividing the GSH value by the GSSG value. The diacron-reactive oxygen metabolite (d-ROM) test was used to assess lipid peroxidation ${ }^{(18)}$, and was performed according to the manufacturer's instructions (Diacron International, Grosseto, Italy). The analysis was fully automated using a Technicon RA 1000 system (Technicon Instruments Corporation, Tarrytown, NY, USA). The specificity and 
sensitivity of the d-ROM test used to measure oxidative stress have been questioned in sports medicine ${ }^{(19,20)}$. However, the d-ROM test has been validated by electron paramagnetic resonance spectrometry, which is considered as the golden standard method to measure the oxidation-reduction phenomena in vitro ${ }^{(21)}$. Moreover, the d-ROM test has also been validated successfully in large populations of healthy subjects, including athletes, and is regarded as a reproducible method for the quantitative evaluation of the peroxidation of organic compounds ${ }^{(22-24)}$.

In the present study, we only measured non-enzymatic endogenous antioxidants. The term 'endogenous defence system' in the present study only refers to the response of uric acid, glutathione (total glutathione; TGSH), cysteine, homocysteine and cysteine-glycine. The thiols, including glutathione (TGSH), cysteine, homocysteine and cysteine-glycine, were analysed in the plasma by chemical reduction and by using the 'homocysteine by HPLC' kit provided by Biorad Laboratories GmbH (Munich, Germany). Total thiols represent the sum of glutathione, cysteine, homocysteine and cysteine-glycine. The endogenous antioxidant uric acid was analysed in the plasma with the standard routine measurements using a Modular $\mathrm{P}^{\circledR}$ Analyser (Hitachi, Tokyo, Japan). Ferric reducing/antioxidant power was determined in the plasma as described elsewhere ${ }^{(25)}$. For the determination of $\alpha$-tocopherol and total tocopherol by HPLC, proteins were precipitated by the addition of three volumes of isopropanol, followed by centrifugation at $3000 \mathrm{~g}$ at $4^{\circ} \mathrm{C}$ for $15 \mathrm{~min}$. The internal standard tocol was added to isopropanol, and $5 \mu \mathrm{l}$ of the clear supernatant were used for the analysis ${ }^{(26)}$. A fluorescence detector that operated at 295 (excitation) and 330 (emission) was used for the detection with a CV below $5 \%$. Standards prepared in $1 \%$ bovine serum albumin in PBS were used for quantification. For the determination of ascorbic acid (AA) in the plasma, heparinised plasma was immediately acidified using an equal volume of $10 \%$ meta-phosphoric acid, and was stored at $-70^{\circ} \mathrm{C}$ until analysis within 3 months. Samples were analysed by $\operatorname{HPLC}^{(27,28)}$ with a CV below $5 \%$. Plasma calibrators quantified against the NIST 970 SRM served as the standards. For the analysis of total polyphenols, $50 \mu \mathrm{l}$ of heparinised plasma were mixed with $150 \mu \mathrm{l}$ of ethanol for $2 \mathrm{~min}$ before centrifugation at $3000 \mathrm{~g}$ at $4^{\circ} \mathrm{C}$ for $15 \mathrm{~min}$. Fifty microlitres of the clear supernatant were used for the Folin-Ciocalteu method as described previously $^{(29)}$. Quercetin prepared in ethanol served as a standard solution, and the results are given as $\mu \mathrm{mol} / \mathrm{l}$ quercetin equivalents with a CV below $10 \%$. Total carotenoids are the sum of lutein, zeaxanthin, $\beta$-cryptoxanthin, $\alpha$-carotene, $\beta$-carotene and lycopene. They were determined in the plasma by HPLC. Proteins were precipitated and removed by the addition of a 4.5 volume of isopropanol followed by centrifugation at $3000 \mathrm{~g}$ at $4^{\circ} \mathrm{C}$ for $15 \mathrm{~min}$. The internal standard astaxanthin was added to isopropanol. Twenty-five microlitres of the clear supernatant were used for the analysis. The mobile phases consisted of A: $20 \%$ water and $24 \%$ acetone in ethanol and $\mathrm{B}$ : acetone. The gradient conditions were as follows: from 2 to $100 \%$ B within $20 \mathrm{~min}$ followed by $100 \%$ B for $15 \mathrm{~min}$. Detection was performed at $453 \mathrm{~nm}$ using a variable wavelength detector. Plasma calibrators quantified against the NIST 968c SRM were used as the standards, and the CV for the method was below $5 \%$.

\section{Statistical analyses}

A two-way repeated-measures ANOVA for normally distributed data was used to study the changes in several parameters over time in the two groups, and to compare the amplitude of the changes before and after the first and second games. Where significant time differences were found, Dunnett's post hoc test (to compare data at individual time points to baseline values) or Tukey's post hoc test was applied. Data that were not normally distributed were analysed using non-parametric Friedman's test with Dunn's post hoc test. $P$ values below 0.05 were considered statistically significant. Data are presented as means and standard error of the mean (SE). The Statistical Package for the Social Sciences (version 12.0; SPSS, Inc., Chicago, IL, USA) and Statistica (version 7.0; StatSoft, Inc., Tulsa, OK, USA) were used for the analyses.

\section{Results}

\section{Effects of active recovery training}

No differences were observed between the active and passive recovery groups for the levels of endogenous and dietary antioxidants or oxidative stress markers. However, there was a significant time effect on the response of both endogenous and dietary antioxidants as well as on oxidative stress markers. Therefore, changes observed after the games are presented as a mean for all sixteen players in Figs. 1(a-c) and 2(a-d).

\section{Oxidative stress markers}

GSSG significantly increased with a similar magnitude after the first (30 (SE 9)\%) and second games (42 (SE 9) \%) (Fig. 1(a)), whereas GSH remained unchanged after both the games. However, GSH decreased at $45 \mathrm{~h}$ (-25 (SE 5) \%) and $69 \mathrm{~h}(-20(\mathrm{SE} 8) \%)$ after the first game, and thus the pre-second game GSH levels were lower than the pre-first game levels (Table 1). As a result of increased GSSG and unchanged GSH, the GSH:GSSG ratio decreased after both the games. However, the decrease in the GSH:GSSG ratio was statistically significant only after the first game $(-13$ (SE 7) \%). The GSH:GSSG ratio returned to baseline $21 \mathrm{~h}$ after the first game, and significantly decreased below baseline at 45 and $69 \mathrm{~h}$. Consequently, the GSH:GSSG ratio remained unchanged after the second game, possibly due to low pre-game values of GSH (Table 1). Lipid peroxidation measured by the d-ROM test was not altered acutely during the games, and remained unchanged at all time points, except at $69 \mathrm{~h}$, when it was 7 (SE 2) \% lower than the baseline values (Table 1).

\section{Endogenous (non-enzymatic) antioxidant compounds}

Fig. 1(b and c) shows changes in TGSH and total thiols during the first and second games. The general response in the endogenous antioxidants was characterised by a robust increase immediately after both the games. More specifically, both TGSH (Fig. 1(b)) and uric acid increased with similar magnitude after both the games. Ferric reducing/antioxidant power also increased similarly after both the games (Table 2). Although the magnitude of change for cysteine, 

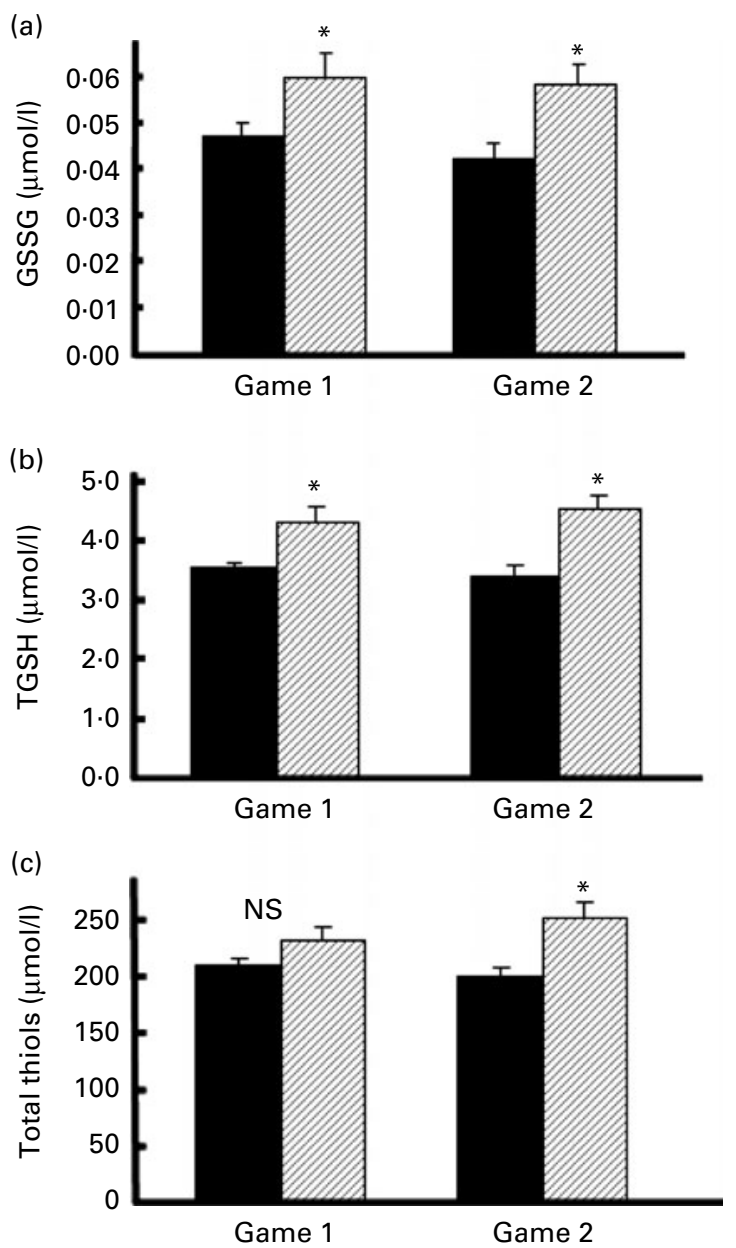

Fig. 1. (a-c) Pre-game ( $\square$ ) and immediate post-game ( $($ ) values for GSSG, total glutathione (TGSH) and total thiols following two soccer games separated by $72 \mathrm{~h}$ in elite female players $(n 16)$. ${ }^{*}$ Mean values were significantly higher than the pre-game values $(P<0.05)$.

cysteine-glycine and total thiols was comparable following both the games, the increase in these compounds reached a statistically significant level only after the second game (Table 2).

\section{Dietary antioxidants}

Fig. 2(a-d) shows the acute changes in total tocopherols, total polyphenols, total carotenoids and AA before and after the games. The dietary antioxidant response pattern was characterised by either a rapid and persistent increase ( $\alpha$-tocopherol, total tocopherol and AA), decrease (polyphenols) or a delayed increase (carotenoids) after the first game. $\alpha$-Tocopherol, total tocopherols and AA (Fig. 2(a) and (d)) increased immediately after the first game, and remained elevated at all time points, except for a temporary normalisation of AA at $21 \mathrm{~h}$. Consequently, the pre-second game levels of the dietary antioxidants AA, $\alpha$-tocopherol and total tocopherol were significantly higher than pre-first game levels. Immediately after the second game, there were no further significant increases in total tocopherols and AA (Fig. 2(a) and (d)), whereas $\alpha$-tocopherol slightly decreased (Table 3 ). The total carotenoid compounds are not involved in the acute antioxidant response to exercise, as there were no immediate changes in carotenoids after both the first and the second games (Fig. 2(c)). The increase in carotenoids was delayed and occurred several hours after the first game: lutein, zeaxanthin and $\beta$-cryptoxanthin increased at $21 \mathrm{~h}$, and lycopene, $\alpha$-carotene and $\beta$-carotene increased only at $69 \mathrm{~h}$ after the first game (Table 3). Consequently, the pre-second game levels of the carotenoids lutein, $\beta$-cryptoxanthin, $\alpha$-carotene, and lycopene and total carotenoids were significantly higher than pre-first game levels. Finally, the level of total polyphenols significantly decreased immediately after the first game, remained reduced until $69 \mathrm{~h}$ and was not affected by the second game (Fig. 2(b)).
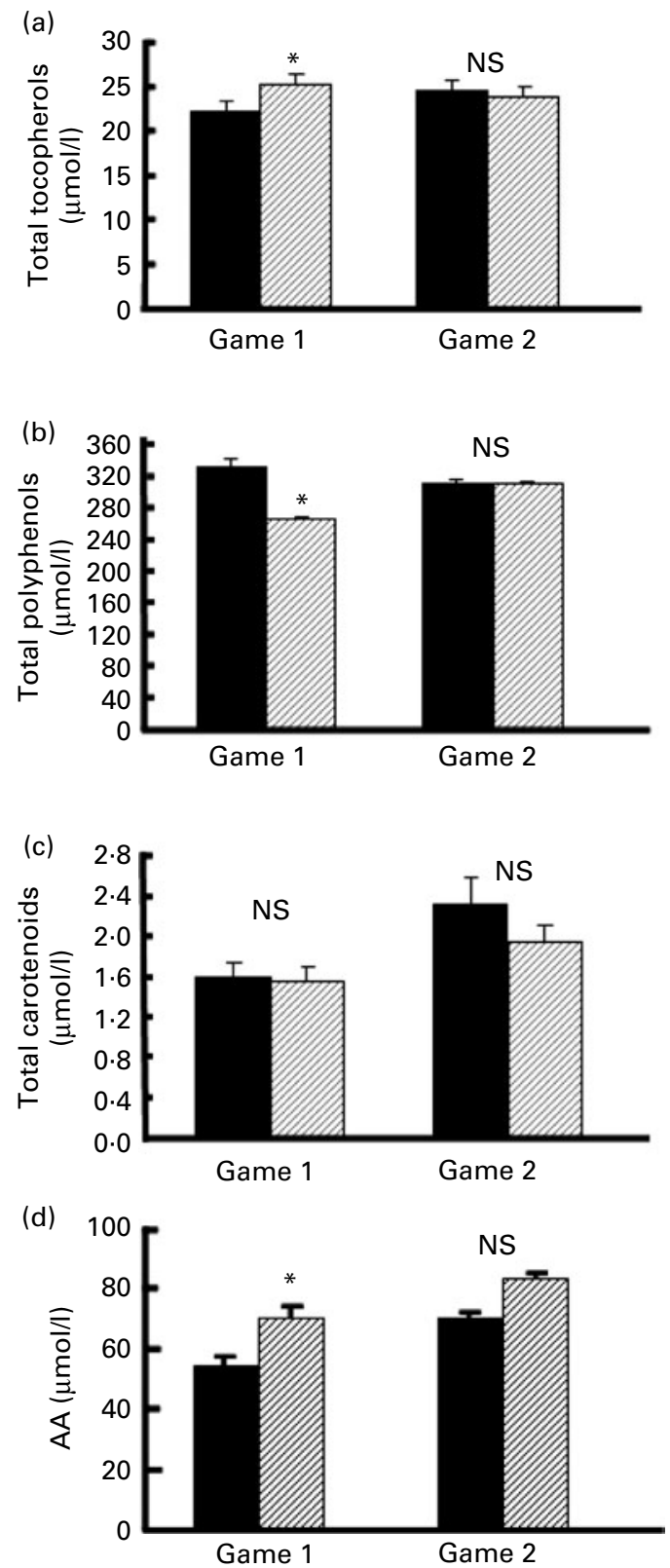

Fig. 2. (a-d) Pre-game ( $\square$ ) and immediate post-game ( $\square$ ) values for the dietary antioxidants total tocopherols, total polyphenols, total carotenoids and ascorbic acid (AA) following two soccer games separated by $72 \mathrm{~h}$ in elite female players $(n 16)$. ${ }^{*}$ Mean values were significantly different from the pre-game values $(P<0.05)$. 
Table 1. Oxidative stress markers and GSH response after two soccer games separated by $72 \mathrm{~h}$ active or passive recovery (Mean values with their standard errors)

\begin{tabular}{|c|c|c|c|c|c|c|c|c|c|c|c|c|c|c|c|}
\hline \multirow{2}{*}{$\begin{array}{l}\text { Oxidative stress markers } \\
(\mu \mathrm{mol} / \mathrm{l})\end{array}$} & \multicolumn{2}{|c|}{$\begin{array}{c}\text { Baseline } \\
(-3 h)\end{array}$} & \multicolumn{2}{|c|}{$\begin{array}{l}\text { IP game } 1 \\
(0 \mathrm{~h})\end{array}$} & \multicolumn{2}{|c|}{$\begin{array}{l}\text { Day } 2 \\
(21 \mathrm{~h})\end{array}$} & \multicolumn{2}{|c|}{$\begin{array}{l}\text { Day } 3 \\
(45 \mathrm{~h})\end{array}$} & \multicolumn{2}{|c|}{$\begin{array}{l}\text { Pre-game } 2 \\
\quad(69 \mathrm{~h})\end{array}$} & \multicolumn{2}{|c|}{$\begin{array}{l}\text { IP game } 2 \\
(72 \mathrm{~h})\end{array}$} & \multicolumn{3}{|c|}{ ANOVA } \\
\hline & Mean & SE & Mean & SE & Mean & SE & Mean & SE & Mean & SE & Mean & SE & $G$ & $T$ & $G \& T$ \\
\hline $\mathrm{GSH}(n 16)$ & 1.9 & 0.1 & $2 \cdot 0$ & 0.2 & $2 \cdot 0$ & 0.2 & $1.4 \dagger$ & 0.1 & $1.5 \dagger$ & 0.1 & 1.7 & 0.1 & & $x$ & \\
\hline GSH:GSSG ratio $(n 16)$ & 41 & 3 & $35 t$ & 3 & 37 & 3 & $30 \dagger$ & 2 & $34 \dagger$ & 2 & $29 \dagger$ & 2 & & $x$ & \\
\hline $\begin{array}{l}\text { d-ROM } \\
\quad(\text { CARR. U) }(n 16)\end{array}$ & 245 & 25 & 249 & 25 & 244 & 24 & 246 & 24 & $225 \dagger$ & 21 & 240 & 22 & & $x$ & \\
\hline
\end{tabular}

$I P$, immediately post game; $G$, significant effect of recovery group; $T$, significant time effect; G\& $T$, significant interaction effect between the two ANOVA factors; CARR. U, Carratelli units.

$\dagger$ Mean values were significantly lower than the pre-game values $(P<0.05)$.

\section{Discussion}

In the present study, we established for the first time the response pattern of oxidative stress markers and antioxidant compounds during two repeated elite female soccer games separated by $72 \mathrm{~h}$ active or passive recovery. The main findings were that similar acute oxidative stress and endogenous antioxidant responses and dissimilar dietary antioxidant responses occur after two repeated elite female soccer games. Furthermore, the dietary antioxidant response, but not the endogenous antioxidant response, to a female soccer game is persistent. Combined endogenous and dietary antioxidant defences seem to prevent lipid peroxidation during two repeated elite female soccer games. Finally, the present findings do not support the beneficial role of active recovery training in the antioxidant response in elite female soccer players.

The active recovery training consisting of cycling $(60 \%$ peak heart rate) and resistance training $(<50 \% 1$ repetition maximum) has no influence on the recovery of oxidative stress markers or antioxidant levels during repeated soccer games in elite female players. The present findings are in accordance with our previous data suggesting the lack of effects of active recovery training on the recovery pattern of specific neuromuscular fatigue parameters, blood markers of physical stress ${ }^{(7)}$ and inflammatory markers ${ }^{(13)}$. In accordance with the present results, recovery strategies such as active recovery or cold water immersion do not accelerate the recovery of several neuromuscular or biochemical markers following intermittent exercise ${ }^{(30-33)}$. Furthermore, the game performance (measured as mean $\mathrm{HR}$ and time $>85$ HRpeak) was similar in both the games, and did not differ between the players in the active or passive recovery group. Regardless of the recovery strategy, the players were able to perform at a high intensity during the second game. This further supports the finding of a lack of effect of active recovery training on any biological marker used in the present study.

Increases in GSSG are associated with exercise intensity ${ }^{(34)}$, and the low-intensity recovery training in the time period after the first game did not affect the accumulation of GSSG. Importantly, although the effects of active recovery training have been evaluated using a large battery of physiological and biological parameters ${ }^{(7,13)}$, the possibility that this recovery strategy might have affected other parameters not evaluated in the present study cannot be excluded.

The main finding of the present study was that the exercise intensity of both the games mediated oxidation of GSH, thereby causing increased GSSG levels. The oxidation of GSH to GSSG is a sensitive marker of free radical production since GSH donates a pair of hydrogen ions to neutralise peroxides $^{(35)}$. When the oxidation of GSH to GSSG exceeds the enzymatic reducing capacity, GSSG is exported from

Table 2. Endogenous antioxidant and ferric reducing/antioxidant power (FRAP) response after two soccer games separated by $72 \mathrm{~h}$ active or passive recovery

(Mean values with their standard errors)

\begin{tabular}{|c|c|c|c|c|c|c|c|c|c|c|c|c|c|c|c|}
\hline \multirow{2}{*}{$\begin{array}{l}\text { Endogenous } \\
\text { antioxidant } \\
\text { compounds } \\
(\mu \mathrm{mol} / \mathrm{l})\end{array}$} & \multicolumn{2}{|c|}{$\begin{array}{c}\text { Baseline } \\
(-3 h)\end{array}$} & \multicolumn{2}{|c|}{$\begin{array}{l}\text { IP game } 1 \\
(0 \mathrm{~h})\end{array}$} & \multicolumn{2}{|c|}{$\begin{array}{l}\text { Day } 2 \\
(21 \mathrm{~h})\end{array}$} & \multicolumn{2}{|c|}{$\begin{array}{l}\text { Day } 3 \\
(45 \mathrm{~h})\end{array}$} & \multicolumn{2}{|c|}{$\begin{array}{l}\text { Pre-game } 2 \\
\quad(69 \mathrm{~h})\end{array}$} & \multicolumn{2}{|c|}{$\begin{array}{l}\text { IP game } 2 \\
(74 \mathrm{~h})\end{array}$} & \multicolumn{3}{|c|}{ ANOVA } \\
\hline & Mean & SE & Mean & SE & Mean & SE & Mean & SE & Mean & SE & Mean & SE & $G$ & $T$ & $G \& T$ \\
\hline Cys $(n 16)$ & 180 & 4 & 200 & 10 & 184 & 6 & 183 & 5 & 172 & 8 & $217^{*}$ & 12 & & $\times$ & \\
\hline $\begin{array}{l}\text { Homocysteine } \\
\qquad(n 16)\end{array}$ & $9 \cdot 1$ & $1 \cdot 2$ & $9 \cdot 2$ & $1 \cdot 0$ & $8 \cdot 8$ & 1.0 & $9 \cdot 0$ & $1 \cdot 2$ & $8 \cdot 1$ & 1.0 & 9.4 & $1 \cdot 1$ & & $\times$ & \\
\hline Cys-Gly ( $n$ 16) & $17 \cdot 3$ & 0.7 & $19 \cdot 7$ & $2 \cdot 0$ & $17 \cdot 6$ & 0.6 & $17 \cdot 4$ & 0.7 & $15 \cdot 6$ & 0.9 & $20 \cdot 4^{*}$ & $1 \cdot 2$ & & $x$ & \\
\hline Uric acid (n 16) & 246 & 14 & $273^{\star}$ & 15 & 259 & 12 & 257 & 11 & 253 & 10 & $288^{*}$ & 11 & & $\times$ & \\
\hline $\operatorname{FRAP}(n 16)$ & 1450 & 39 & $1588^{*}$ & 39 & 1416 & 34 & 1379 & 31 & 1360 & 40 & $1484^{*}$ & 48 & & $x$ & \\
\hline
\end{tabular}

IP, immediately post game; $G$, significant effect of recovery group; $T$, significant time effect; $G \& T$, significant interaction effect between the two ANOVA factors.

${ }^{*}$ Mean values were significantly higher than the pre-game values $(P<0.05)$. 
Table 3. Dietary antioxidant response after two soccer games separated by $72 \mathrm{~h}$ active or passive recovery (Mean values with their standard errors)

\begin{tabular}{|c|c|c|c|c|c|c|c|c|c|c|c|c|c|c|c|}
\hline \multirow{2}{*}{$\begin{array}{l}\text { Dietary antioxidant } \\
\text { compounds }(\mu \mathrm{mol} / \mathrm{l})\end{array}$} & \multicolumn{2}{|c|}{$\begin{array}{l}\text { Baseline } \\
(-3 h)\end{array}$} & \multicolumn{2}{|c|}{$\begin{array}{l}\text { IP game } 1 \\
(0 h)\end{array}$} & \multicolumn{2}{|c|}{$\begin{array}{l}\text { Day } 2 \\
(21 \mathrm{~h})\end{array}$} & \multicolumn{2}{|c|}{$\begin{array}{l}\text { Day } 3 \\
(45 \mathrm{~h})\end{array}$} & \multicolumn{2}{|c|}{$\begin{array}{l}\text { Pre-game } 2 \\
\quad(69 \mathrm{~h})\end{array}$} & \multicolumn{2}{|c|}{$\begin{array}{l}\text { IP game } 2 \\
(74 \mathrm{~h})\end{array}$} & \multicolumn{3}{|c|}{ ANOVA } \\
\hline & Mean & SE & Mean & SE & Mean & SE & Mean & SE & Mean & SE & Mean & SE & $G$ & $T$ & $G \& T$ \\
\hline$\alpha$-Tocopherol $(n+16)$ & $20 \cdot 2$ & $1 \cdot 1$ & $23 \cdot 0^{*}$ & 1.2 & $22 \cdot 4^{*}$ & $1 \cdot 2$ & $21 \cdot 8^{*}$ & 0.9 & $22 \cdot 1^{*}$ & $1 \cdot 1$ & 21.4 & 1.1 & & $x$ & \\
\hline Lutein $(n 16)$ & 0.18 & 0.02 & 0.19 & 0.02 & $0.23^{\star}$ & 0.03 & $0.22^{*}$ & 0.03 & $0.21^{*}$ & 0.02 & 0.17 & 0.02 & & $x$ & \\
\hline Zeaxanthin $(n$ 16) & 0.04 & 0.00 & 0.04 & 0.00 & $0.06^{*}$ & 0.01 & 0.05 & 0.00 & 0.04 & 0.00 & 0.03 & 0.00 & & $x$ & \\
\hline Lycopene $(n 16)$ & 0.56 & 0.04 & 0.53 & 0.04 & 0.65 & 0.08 & 0.71 & 0.08 & $0.98^{\star}$ & 0.12 & $0.77^{\star}$ & 0.05 & & $x$ & \\
\hline$\beta$-Cryptoxanthin $(n 16)$ & 0.18 & 0.03 & 0.19 & 0.04 & $0.23^{*}$ & 0.06 & 0.21 & 0.04 & $0.24^{\star}$ & 0.04 & 0.20 & 0.03 & & $x$ & \\
\hline$\alpha$-Carotene $(n 16)$ & 0.13 & 0.02 & 0.12 & 0.02 & 0.15 & 0.04 & 0.16 & 0.03 & $0 \cdot 18^{*}$ & 0.03 & 0.14 & 0.02 & & $x$ & \\
\hline$\beta$-Carotene $(n 16)$ & 0.51 & 0.06 & 0.49 & 0.06 & 0.58 & 0.10 & 0.59 & 0.09 & $0.67^{\star}$ & 0.10 & $0.64^{*}$ & 0.07 & & $x$ & \\
\hline
\end{tabular}

IP, immediately post game; $G$, significant effect of recovery group; $T$, significant time effect; $G \& T$, significant interaction effect between the two ANOVA factors.

${ }^{*}$ Mean values were significantly higher than the pre-game values $(P<0.05)$.

the muscle cells (as well as from other cells such as liver cells) to maintain the GSH:GSSG ratio in the plasma. Thus, the increased GSSG and the reduced GSH:GSSG ratio observed after both the games imply that free radical production was enhanced. The increase in GSSG is similar to what has been observed in several studies following exercise ${ }^{(36,37)}$. It has been suggested that increased levels of free radicals during exercise may cause reduced force production ${ }^{(17)}$. We have shown previously that several neuromuscular parameters were reduced with similar amplitude after both the games ${ }^{(7)}$. Thus, the increased GSSG may be related to the observed neuromuscular fatigue after the games. However, we did not find a significant correlation between changes in CK levels and GSSG after the games.

Interestingly, we observed an increase in GSSG occurring despite unchanged GSH levels. This result can be explained by adequate GSH availability and possible replenishment from other compartments such as the liver. Similarly, an increase in blood GSSG without a concomitant decrease in GSH has been shown previously following exercise ${ }^{(34,38)}$. Although GSSG increased, lipid peroxidation measured by d-ROM test remained unchanged after the two games. This finding implies that antioxidant defence systems in welltrained female players can prevent lipid peroxidation during two repeated soccer games separated by $72 \mathrm{~h}$ recovery. Nevertheless, it is important to highlight the difficulties in detecting reactive intermediates directly in vivo because of their short half-lives ${ }^{(39)}$. The $\mathrm{d}-\mathrm{ROM}$ test has been questioned $^{(19,20)}$, and similar to other methods, it has its advantages and disadvantages, which is why no single measurement can adequately describe oxidative damage ${ }^{(2)}$. It is therefore suggested that the use of a battery of measurements is important to reliably monitor changes in oxidative stress ${ }^{(40)}$. The use of several biomarkers allowed us to highlight the fact that despite the lack of change in lipid peroxidation measured by d-ROM test, increases in GSSG and decreases in the GSSG:GSH ratio together with increases in antioxidant levels occurred.

We observed that the soccer-induced increase in ROS was accompanied by a robust response in several antioxidant compounds after the games. In general, there was a similar acute response pattern of endogenous antioxidants following both the games. This implies that the involvement of the endogenous antioxidants in the early line of defence against ROS is maintained during two repeated soccer games. Our hypothesis is supported by the fact that uric acid and TGSH, both regarded as important plasma antioxidants, increased similarly following both the games. We also observed increases in cysteine and cysteine-glycine after the games. The majority of GSH is synthesised in the liver, and its release during exercise is promoted by increased levels of catecholamines, glucagon and vasopressin ${ }^{(41,42)}$. In the kidney, GSH is metabolised to glutamate, cysteine and glycine, which are subsequently released into the circulation ${ }^{(43)}$. Thus, the increase in cysteine and cysteine-glycine after both the games further implies increased liver GSH turnover. However, although cysteine, cysteine-glycine and total thiols increased following both the games, the amplitude of changes was statistically significant only in response to the second game. This might be explained by the lower pre-second game levels of thiols. Reduced thiol levels can be related to the progressive elevation of dietary antioxidants after the first game, which might have strengthened the total antioxidant defence capacity. In these circumstances, the contribution of thiols to the total antioxidant defence capacity would be less important.

In general, compared with the endogenous antioxidants, the dietary antioxidants showed either a rapid and persistent increase ( $\alpha$-tocopherol, total tocopherols and AA), decrease (polyphenols) or a delayed increase (carotenoids) after the first game. The acute responses of the dietary antioxidants tocopherols, AA and polyphenols were dissimilar following the two repeated soccer games, as the acute increase in tocopherols and AA and decrease in polyphenols observed after the first game did not occur after the second game. The acute increase in the tocopherols and AA and decrease in polyphenols after the first game indicate a role of dietary antioxidants in the early line of defence against increased ROS production. However, contrary to the rapid normalisation of the endogenous antioxidants, there was a persistent elevation in AA and tocopherols and reduction of polyphenols after the first game. AA and tocopherol compounds are provided by diet and stored in the adipose tissue, and only moderate amounts of the total body pool are found in the circulation. The delayed increase in these compounds following the first game suggests that the soccer-induced elevation in ROS may mediate their relocation in order to 
strengthen the endogenous antioxidant defence and restore the redox balance. This response occurred without alteration of the intake of antioxidants during the study period.

Thus, the long-lasting changes in the dietary antioxidant compounds suggest that they are involved in the stabilisation and maintenance of homoeostasis in the pro-oxidant and antioxidant redox balance several days following the first soccer game. These persistent changes might explain the fact that these compounds did not increase further immediately after the second game, and suggest sufficient levels of available dietary antioxidants in the circulation during the second soccer game. We have also reported the lack of acute changes in carotenoids following the soccer games. This is similar to the findings reported previously after intermittent exercise in male subjects ${ }^{(9)}$, suggesting that carotenoids are not involved in the acute response to increased ROS after exercise. However, we observed a delayed increase in carotenoids that started $21 \mathrm{~h}$ after the first game. This recruitment pattern suggests that carotenoids are slowly mobilised from the adipose tissue possibly to strengthen the antioxidant defence capacity in response to the soccer game.

We have shown previously that the two repeated soccer games, with comparable workload, induce similar reductions in sprint ability, isokinetic knee strength, and elevations in $\mathrm{CK}$, urea and perceived muscle soreness ${ }^{(7)}$. The present study reveals that the endogenous antioxidants are similarly recruited during repeated soccer games, whereas there are dissimilarities in the acute response of dietary antioxidants. Data on changes in oxidative stress and antioxidant markers in response to repeated soccer games in male and female players are scarce. However, in line with the present findings, two repeated cycling exercise bouts in males showed similar changes in blood GSH, GSSG, plasma AA, CK, perceived exertion and lipid peroxidation ${ }^{(44,45)}$. In contrast, following repeated eccentric exercise sessions in untrained females, blunted $\mathrm{CK}$, oxidative stress and antioxidant responses occurred during the second exercise session, a phenomenon labelled as the 'repeated bout effect' $^{\text {(46) }}$. Inconsistencies between these studies can be due to the differences in the exercise protocols and the training status of the subjects ${ }^{(38)}$.

In conclusion, the present study established for the first time the time course of changes in circulating oxidative stress and antioxidant systems during two repeated elite female soccer games. Combined endogenous and dietary antioxidant defences seem to prevent lipid peroxidation during two repeated elite female soccer games. The use of active recovery training in the period between two elite female soccer games has no impact on the oxidative stress and antioxidant systems.

\section{Acknowledgements}

The present work was supported by a grant from Swedish National Centre for Research in Sports (89/06). The study was performed at Norwegian School of Sport Sciences and Olympiatoppen, Oslo, Norway. One of the authors, R. B., has an interest in Vitas AS. All authors have contributed to experimental design, data collection and analysis. The authors would like to thank the players from KIF Örebro DFF and Kolbotn IL.

\section{References}

1. Banerjee AK, Mandal A, Chanda D, et al. (2003) Oxidant, antioxidant and physical exercise. Mol Cell Biochem 253, 307-312.

2. Nikolaidis M, Jamurtas A, Paschalis V, et al. (2008) The effect of muscle-damaging exercise on blood and skeletal muscle oxidative stress: magnitude and time-course considerations. Sports Med 38, 579-606.

3. Reid M, Khawli F \& Moody M (1993) Reactive oxygen in skeletal muscle. III. Contractility of unfatigued muscle. J Appl Physiol 75, 1081-1087.

4. Bangsbo J (1994) Energy demands in competitive soccer. J Sport Sci 12, S5-S12.

5. Ascensão A, Rebelo A, Oliviera E, et al. (2008) Biochemical impact of a soccer match-analysis of oxidative stress and muscle damage markers throughout recovery. Clin Biochem 41, 841-851.

6. Fatouros I, Chatzinikolaou A, Douroudos I, et al. (2009) Time-course of changes in oxidative stress and antioxidant status responses following a soccer game. J Strength Cond Res 24, 1389-1398.

7. Andersson H, Raastad T, Nilsson J, et al. (2008) Neuromuscular fatigue and recovery in elite female soccer: effects of active recovery. Med Sci Sports Exerc 40, 372-380.

8. Kingsley M, Wadsworth D, Kilduff L, et al. (2005) Effects of phosphatidylserine on oxidative stress following intermittent running. Med Sci Sports Exerc 37, 1300-1306.

9. Tauler P, Ferrer M, Sureda A, et al. (2008) Supplementation with an antioxidant cocktail containing coenzyme Q prevents plasma oxidative damage induced by soccer. Eur J Appl Physiol 104, 777-785.

10. Andersson H, Karlsen A, Blomhoff R, et al. (2009) Plasma antioxidant responses and oxidative stress following a soccer game in elite female players. Scand J Med Sci Sports (Epublication ahead of print version 23 August 2009).

11. Barnett A (2006) Using recovery modalities between training sessions in elite athletes: does it help? Sports Med 36, 781-796.

12. Reilly $\mathrm{T} \&$ Ekblom B (2005) The use of recovery methods post-exercise. J Sports Sci 23, 619-627.

13. Andersson H, Bøhn SK, Raastad T, et al. (2009) Differences in the inflammatory plasma cytokine response following two elite female soccer games separated by a 72-h recovery. Scand J Med Sci Sport (Epublication ahead of print version 17 September 2009).

14. Akova B, Sürmen-Gür E, Gür H, et al. (2001) Exercise-induced oxidative stress and muscle performance in healthy women: role of vitamin E supplementation and endogenous oestradiol. Eur J Appl Physiol 84, 141-147.

15. Chung S, Goldfarb A, Jamurtas A, et al. (1999) Effect of exercise during the follicular and luteal phase on indices of oxidative stress in healthy women. Med Sci Sports Exerc 31, 409-413.

16. Maughan R, Burke L \& Coyle E (2004) Food, nutrition and sports performance II. In The International Olympic Committee Consensus on Sports Nutrition, pp. 24-49, 104-129: Routledge New York.

17. Powers S \& Jackson M (2008) Exercise-induced oxidative stress: mechanisms and impact on muscle force production. Physiol Rev 88, 1243-1276.

18. Cesarone M, Belcaro G, Carratelli M, et al. (1999) A simple test to monitor oxidative stress. Int Angiol 18, 127-130.

19. Harma M, Harma M \& Erel O (2006) d-ROMs test detects ceruoplasmin, not oxidative stress. Chest 130, 1276-1277.

20. Erel O (2005) A new automated colorimetric method for measuring total oxidant status. Clin Biochem 38, $1103-1111$. 
21. Alberti A, Bolognini L, Macciantelli D, et al. (2000) The radical cation of $N, N$-diethyl-para-phenylendiamine: a possible indicator of oxidative stress in biological samples. Res Chem Intermed 26, 253-267.

22. Banfi G, Malavazos A, Iorio E, et al. (2006) Plasma oxidative stress biomarkers, nitric oxide and heat shock protein 70 in trained elite soccer players. Eur J Appl Physiol 96, 483-486.

23. Bonina F, Puglia C, Cimino F, et al. (2005) Oxidative stress in handball players: effect of supplementation with a red orange extract. Nutr Res 25, 917-924.

24. Iamele L, Fiocchi R \& Vernocchi A (2002) Evaluation of an automated spectrophotometric assay for reactive oxygen metabolites in serum. Clin Chem Labor Med 40, 673-676.

25. Benzie I \& Strain J (1999) Ferric reducing/antioxidant power assay: direct measure of total antioxidant activity of biological fluids and modified version for simultaneous measurement of total antioxidant power and ascorbic acid concentration. Methods Enzymol 299, 15-27.

26. Richheimer S, Kent M \& Bernart M (1994) Reversed-phase high-performance liquid chromatographic method using a pentafluorophenyl bonded phase for analysis of tocopherols. J Chrom A 677, 75-80.

27. Karlsen A, Blomhoff R \& Gundersen T (2005) High-throughput analysis of vitamin $\mathrm{C}$ in human plasma with the use of HPLC with monolithic column and UV-detection. $J$ Chromatogr $B$ Analyt Technol Biomed Life Sci 824, 132-138.

28. Karlsen A, Blomhoff R \& Gundersen T (2007) Stability of whole blood and plasma ascorbic acid. Eur J Clin Nutr 61, $1233-1236$.

29. Maskarinec G, Chan C, Meng L, et al. (1999) Exploring the feasibility and effects of a high-fruit and -vegetable diet in healthy women. Cancer Epidemiol Biomarkers Prev 8, 919-924.

30. Gill ND, Beaven CM \& Cook C (2006) Effectiveness of post-match recovery strategies in rugby players. $\mathrm{Br} J$ Sports Med 40, 260-263.

31. Rowsell G, Coutts A, Reaburn P, et al. (2009) Effects of cold-water immersion on physical performance between successive matches in high-performance junior male soccer players. J Sport Sci 27, 565-573.

32. Tessitore A, Meeusen R, Pagano R, et al. (2008) Effectiveness of active versus passive recovery strategies after futsal games. Strength Cond Res 22, 1402-1412.
33. Kinugasa T \& Kilding A (2009) A comparison of post-match recovery strategies in youth soccer players. $J$ Strength Cond Res 23, 1402-1407.

34. Ji LL, Fu R \& Mitchell EW (1992) Glutathione and antioxidant enzymes in skeletal muscle: effects of fiber type and exercise intensity. J Appl Physiol 73, 1854-1859.

35. Sen CK (1999) Glutathione homeostasis in response to exercise training and nutritional supplements. Mol Cell Biochem 196, $31-42$.

36. Goldfarb A, McKenzie M \& Bloomer R (2007) Gender comparisons of exercise-induced oxidative stress: influence of antioxidant supplementation. Appl Physiol Nutr Metab 32, 1124-1131.

37. Paschalis V, Nikolaidis MG, Fatouros IG, et al. (2007) Uniform and prolonged changes in blood oxidative stress after muscledamaging exercise. In Vivo 21, 877-883.

38. Kyparos A, Vrabas IS, Nikolaidis MG, et al. (2009) Increased oxidative stress blood markers in well-trained rowers following two thousand-meter rowing ergometer race. J Strength Cond Res 23, 1418-1426.

39. Urso M \& Clarkson P (2003) Oxidative stress, exercise and antioxidant supplementation. Toxicology 189, 41-54.

40. Halliwell B \& Whiteman M (2004) Measuring reactive species and oxidative damage in vivo and in cell culture: how should you do it and what do the results mean? Br J Pharmacol 142, 231-255.

41. Lu S, Garcia-Ruiz C, Kulenkamp J, et al. (1990) Hormonal regulation of glutathione efflux. $J$ Biol Chem 265, $16088-16095$

42. Sies H \& Graf P (1985) Hepatic thiol and glutathione efflux under the influence of vasopressin, phenylephrine and adrenaline. Biochem J 226, 545-549.

43. Ji L (2000) Free radicals and antioxidants in exercise and sports. In Exercise and Sport Science, pp. 299-317 [W Garret and D Kirkendal, editors]. Philadelphia, PA: Lippincott Williams \& Wilkins.

44. Viguie CA, Frei B, Shigenaga MK, et al. (1993) Antioxidant and indexes of oxidative stress during consecutive days of exercise. J Appl Physiol 75, 566-572.

45. Bloomer R, Falvo M, Fry A, et al. (2006) Oxidative stress response in trained men following repeated squats or sprints. Med Sci Sports Exerc 38, 1436-1442.

46. Nikolaidis M, Paschalis V, Giakas G, et al. (2007) Decreased blood oxidative stress after repeated muscle-damaging exercise. Med Sci Sports Exerc 39, 1080-1089. 